the variation in urinary PEA levels among individual patients is large.

The above report confirms previous findings of decreased urinary levels of PEA in children with ADHD (Zametkin AJ et al. 1984; Baker GB et al. 1991). An abnormality in the absorption or transportation of phenylalanine is suggested as one explanation for the decreased PEA excretion, but symptoms of ADHD are not improved by treatment with phenylalanine (Zametkin AJ et al. 1987). Monoamines including HVA and 5-HIAA have been linked to ADHD in some studies, but the present report does not support an association. Further investigations are warranted.

\title{
TRIAL OF DIVALPROEX FOR BIPOLAR DISORDER
}

The safety and effectiveness of divalproex sodium (Depakapote ${ }^{\circledR}$ ) in the treatment of 40 children and adolescents, aged 7 to 19 years, with a primary diagnosis of bipolar disorder were evaluated by open-label study (2-8 weeks) at the University of Texas, Galveston; University of Pennsylvania, Philadelphia; SUNY Stonybrook, NY; Massachusetts General Hospital, Boston; and University of Texas, San Antonio. Six subjects (15\%) had comorbid ADHD that required stimulant therapy in addition, and $23(58 \%)$ had a comorbid psychiatric diagnosis that was treated with concurrent medications, including lithium, haloperidol, or lorazepam. A greater than $50 \%$ improvement was obtained in 22 subjects (61\%) as measured by the Mania Rating Scale (MRS). Mean scores of all efficacy measures showed significant improvements $(\mathrm{p}<.001)$ from baseline, including the MRS, Manic Syndrome Scale, Behavior and Ideation Scale, Psychiatric Rating Scale, and Hamilton Rating Scale. At the completion of the study, the mean divalproex dose was $17.5 \mathrm{mg} / \mathrm{kg}$ per day, and the mean serum valproate level was $83.4 \mathrm{mcg} / \mathrm{ml}$. Twenty seven subjects (68\%) reported one or more adverse events, the most common including headache (7), nausea (7), vomiting (6), diarrhea (4), and somnolence (4). Twenty four subjects discontinued treatment because of lack of efficacy (6), drug intolerance (6), noncompliance (6), and other reasons (6). None required drug withdrawal because of abnormal laboratory values. A planned double-blind, placebo-controlled study to follow the open-label period was abandoned because of insufficient number of patients. (Wagner KD, Weller EB, Carlson GA et al. An open-label trial of divalproex in children and adolescents with bipolar disorder. IAm Acad Child Adolesc Psychiatry October 2002;41:12241230). (Reprints: Dr Wagner, Department of Psychiatry and Behavioral Sciences, University of Texas Medical Branch, 301 University Blvd, Galveston, TX 77555).

COMMENT. The authors conclude that the results provide support for the safety and effectiveness of divalproex in the treatment of bipolar disorder in children and adolescents. However, failure to complete the study period in more than $50 \%$ of patients is not a favorable recommendation.

Tha comorbidity of ADHD in children with bipolar disorder is a frequent occurrence, with estimates up to $94 \%$ (Wozniak et al. 1995). Treatment often requires a combination of stimulant medications and antidepressants.

\section{NEUROMUSCULAR DISORDERS}

\section{TICK PARALYSIS}

Six children aged 3.3 to 5.5 years ( 5 girls and 1 boy), of 26 admitted with acute muscle weakness to the University of Mississippi Medical Center, Jackson, over a 5 year period (1992-97), were diagnosed with tick paralysis. The initial 
diagnosis in 3 of the 6 children was Guillain-Barre syndrome, the clinical and electrodiagnostic findings being indistinguishable from tick paralysis. Treatment with IV immunoglobulin was not of benefit, and the diagnosis of tick paralysis was established by finding a tick on the scalp or nape of the neck, and a rapid recovery within 24 hours following its removal. Clinical findings included leg, arm, and trunk weakness (6), facial weakness (4), bulbar weakness (1), eye muscle weakness (1), respiratory failure (1), and areflexia (5). Cranial nerves were normal in 2 . Vibratory sense was mildly diminished in patients tested. CSF studies were normal in 4 tested. Low CMPA amplitudes found on initial electrodiagnostic studies increased rapidly after removal of the tick and in parallel with clinical improvement. Distal latencies and motor conduction velocities were normal, but the $\mathrm{H}$ reflex was absent. Repetitive nerve stimulation at both slow and fast rates was normal. Sensory nerve action potentials were within normal limits but showed a consistent increase in amplitude on recovery. (Vedanarayanan VV, Evans OB, Subramony SH. Tick paralysis in children. Electrophysiology and possiblity of misdiagnosis. Neurology October (1 of 2) 2002;59:1088-1090). (Reprints: Dr SH Subramony, 2500 North State Street, Department of Neurology, Jackson, MS 39216).

COMMENT. A child admitted with acute, rapidly progressive muscle weakness should be examined for a tick. The incidence of tick paralysis among children admitted with acute muscle weakness is $25 \%$. Unless the tick is considered as the cause, found by examination of scalp and neck, and removed, the clinical and electrodiagnostic findings may be difficult to distinguish from those of Guillain-Barre syndrome, leading to misdiagnosis and inappropriate therapy. The tick causing paralysis in N America is the Dermacentor species, and the illness is milder and shorter than that in Australia, caused by Ixodes holocyclus (Grattan-Smith PJ et al. Brain 1997;120:1975-1987). A toxin is secreted by the female tick salivary gland which is presumed to cause an interference with transmission at motor nerve terminals. Removal of the tick is followed by a rapid recovery. Previous reports have cited Guilain-Barre syndrome in the differential diagnosis (Haller JS, Fabara JA. Am J Dis Child 1972;124:915-917), but the present article emphasizes the similarities of clinical and electrodiagnostic findings. Alternative causes of acute paralysis to be considered include organophosphate pesticide poisoning and shellfish poisoning (Millichap JG. Environmental Poisons in our Food. Chicago, PNB Publ, 1993).

Lyme disease neurologic manifestations. A more common and well known illness and neurologic syndrome caused by tick bite is Lyme disease or Lyme neuroborreliosis. The infecting organism is a spirochete, $B$ burgdorferi, that may be isolated from its vectors, Ixodes scapularis and pacificus, and from a patient's blood, skin lesions, or CSF (AAP Red Book 25th ed. 2000). A Guillain-Barre syndrome can occur with Lyme disease but is rare, reported in only 1 of 96 patients, ages 3 to 19 years, and sometimes atypical, with mild lymphocytic pleocytosis in the CSF as well as the characteristic elevated protein (Belman AL et al. Neurology 1993;43:2609-2614). Most common neurologic symptoms and complications of Lyme disease include headache (71\%), facial palsy (14\%), aseptic meningitis, and pseudotumor cerebri. Meningoradiculitis (Bannwarth's syndrome) and peripheral neuropathy are rare in children and occur more commonly in adult Lyme disease. Diagnosis of neuroborreliosis requires characteristic neurologic signs and either erythema migrans, arthritis or heart involvement, positive spinal fluid serology for anti- $B$ burgdorferi antibodies, or seroconversion (Bingham PM et al. Pediatrics 1995;96:1053-1056). See Progress in Pediatric Neurology III 1997, pp484-5, for review of Lyme disease neurologic 
syndromes.

\section{DEMYELINATING AND DEGENERATIVE DISEASES}

\section{NATURAL HISTORY OF EARLY ONSET MULTIPLE SCLEROSIS}

The clinical course of multiple sclerosis (MS) in 116 patients with onset before age 16 (prevalence $3.6 \%$ in total MS clinic attendees) was evaluated by longitudinal study (mean duration $19.76+/-0.9$ years) at the University of British Columbia MS Clinic. Mean age of onset of MS was $12.73+/-0.25$ years; 23 (19.8\%) had onset at age 10 or younger, and $6(5.2 \%)$ at age 7 or younger. The female to male ratio was 2.87:1. Female preponderance was highest for patients with onset at ages 13 - 14 (pubertal age). Most frequent initial symptoms were sensory disturbances (25.9\%), optic neuritis (21.6\%), brainstem dysfunction (12.9\%), gait disorders (9.4\%), and cerebellar incoordination (6.9\%). Sensory and brainstem disturbances were more frequent in girls, whereas boys were affected more by motor and gait disturbances. The MS course was primary progressive (PP) in 3 cases (2.6\%), and secondary progressive (SP) in 60 (53.1\%), with $50 \%$ probability of SP by 23 years after onset. For relapsing remitting (RR) or SPMS cases, the mean duration from onset to an Expanded Disability Status Scale (EDSS) 3 was 16.03 $+/-1.17$ years, at a mean age of $28.47+/-1.14$ years. EDSS 6 was reached by 19.39 $+/-1.43$ years, at a mean age of $32.32+/-1.44$ years. Annual relapse rate was 0.54 $+/-0.05$ per year. The course of MS was significantly correlated with the number of relapses in the first year after onset. The majority of cases had a RR course, and early age at permanent disability. Disease-modifying therapy should be considered early in the course of early onset MS. (Boiko A, Vorobeychik G, Paty D et al. Early onset multiple sclerosis. A longitudinal study. Neurology October ( 1 of 2) 2002;59:1006-1010). (Reprints: Dr DW Paty, Room S195, 2211 Wesbrook Mall, UBCH, Vancouver, BC V6T 2B5, Canada).

COMMENT. Patients with early onset MS (EOMS) tend to recover from the initial manifestations and have a relatively long first and second remission. However, EOMS cases include patients with frequent relapses, early age at permanent disability, and occurrence of malignant cases. In those with duration more than 10 years, $65 \%$ are disabled. The frequency of relapses in the first 5 years after onset, and the duration of first and second remissions correlate with the risk of permanent disability. Initial brainstem symptoms are predictive of a poor prognosis. Therapy should be considered sooner rather than later in EOMS.

\section{PATTERNS OF REGRESSION IN RETT SYNDROME}

Patterns and features of regression in a case series of 53 girls and women with Rett syndrome were studied at the Institute of Child Health and Great Ormond Street Children's Hospital, London, UK. Diagnostic criteria for classical Rett syndrome were met in 46 cases, and 7 had an atypical/variant form. The most common period for regression was 12 - 18 months, reported by parents or in casenotes in $49 \%$ of patients. Mean age of regression was 16 months. Skills lost in order of frequency were hand use $(85 \%)$, non-verbal vocalizations and simple gestures (59\%), non-verbal play (51\%), motor skills (49\%), and words (45\%). Preregression developmental delays were noted in more than two-thirds of cases ( $85 \%$ in youngest cases when parental reports were most reliable). Age at regression was not an index of neurological severity (epilepsy, breathing abnormalities, mobility, joint contractures, and oral-motor dysfunction). (Charman T, Cass H, Owen L et al. Regression in individuals with Rett syndrome. 